\title{
Multi-functional nano-electronics constructed using boron phosphide and silicon carbide nanoribbons
}

\author{
Jichen Dong ${ }^{1}$, Hui $\mathrm{Li}^{1}$ and $\mathrm{Li}^{\mathrm{Li}^{2}}$ \\ First-principles density functional theory and non-equilibrium Green function calculations provide theoretical support for the \\ promising applications of multi-functional nano-electronics constructed using zigzag boron phosphide (BP) nanoribbons \\ ( $z$ BPNRs) and silicon carbide nanoribbons ( $z$ SiCNRs). The results indicate that $z$ BPNRs are non-magnetic direct bandgap \\ semiconductors with bandgaps of $\sim 1 \mathrm{eV}$. Devices constructed using hybrid $z$ SiC-BP-SiC nanoribbon structures are found to \\ exhibit not only significant field-effect characteristics but also tunable negative differential resistance. Moreover, ' $Y$ '- and \\ ' $\Delta$ '-shaped nano-structures composed of $z B P N R s$ and $z$ SiCNRs exhibit pronounced spin polarization properties at their edges, \\ suggesting their potential use in spintronic applications. Interestingly, a transverse electric field can convert zBPNRs to \\ non-magnetic indirect bandgap semiconductors, ferrimagnetic semiconductors or half-metals depending on the strength \\ and direction of the field. This study may provide a new path for the exploration of nano-electronics. \\ NPG Asia Materials (2013) 5, e56; doi:10.1038/am.2013.31; published online 26 July 2013
}

Keywords: BP nanoribbons; density functional theory; hybrid structure; multi-functional nano-electronics; non-equilibrium Green Function; SiC nanoribbons

\section{INTRODUCTION}

Following the discovery of graphene, considerable research interest has been focused on graphene-based nano-electronics because of the peculiar structure and properties of graphene. ${ }^{1-4}$ According to scaling theory, ${ }^{5}$ ultra-thin graphene is capable of effectively suppressing the short-channel effect of field-effect transistors (FETs), which is one of the largest obstacles in maintaining the high performance of conventional FETs at the nanoscale. In addition, the electron mobility of graphene is exceptionally large, enabling fast-speed atomically thin FETs to be fabricated. ${ }^{1-4,6,7}$ As the zero bandgap of graphene results in very small on-off ratios of graphene FETs and severely constrains its application in logics, ${ }^{2,6,7}$ considerable efforts have been devoted to opening a sizeable bandgap for graphene and/or seeking other semiconducting graphene-like materials. The frequently reported advances have primarily focused on preparing graphene nanoribbons (GNRs), ${ }^{8-10}$ chemically decorating graphene ${ }^{11-12}$ and semiconducting boron nitride sheets, hybrid B-N-C sheets and $\mathrm{MoS}_{2}$ sheets. ${ }^{13-16}$

In addition to FET applications, some graphene devices have been observed to exhibit negative differential resistance (NDR), which can be used in frequency multipliers, memory and oscillators. Theoretical calculations have shown that GNRs with zigzag, armchair or chiral-shaped edges can exhibit NDR if properly treated, such as through the introduction of dopants, vacancies and so on. ${ }^{17-20}$ Dragoman et $a .^{21}$ predicted significant NDR effects in graphene barriers in which the electron transport was dominated by a relativistic-like Dirac equation. Specific Ni/graphene/Ni vertical heterojunctions have been found to exhibit both magneto-resistance and NDR. ${ }^{22}$ Cheraghchi et al. ${ }^{23}$ reported that an odd-even-mediated NDR effect emerged in some GNR-carbon chain-GNR junctions. A slight NDR related to the competition between the conduction of electrons and holes has been observed in a three-terminal graphene FET configuration. ${ }^{24}$

Some evidence for the potential to construct spintronic devices using graphene or other monoatomic layer materials has recently been reported. Theoretical calculations predicted that anti-ferromagnetic semiconducting $z$ GNRs might be changed to half-metals by a transverse electric field. ${ }^{25}$ It has been found that by using a transverse electric field, the bandgaps of non-magnetic semiconducting zigzag boron nitride nanoribbons ( $z$ BNNRs) can be tuned and even closed. ${ }^{26,27}$ Previous investigations have shown that non-magnetic zigzag silicon carbide nanoribbons ( $z$ SiCNRs) are semiconductors with very small bandgaps; ${ }^{28,29}$ while under a transverse electric field, these nanoribbons were observed to convert

\footnotetext{
${ }^{1}$ Ministry of Education, Key Laboratory for Liquid-Solid Structural Evolution and Processing of Materials, Shandong University, Jinan, China and ${ }^{2}$ Faculty of Applied Science and Textiles, The Institute of Textiles and Clothing, The Hong Kong Polytechnic University, Hong Kong, China

Correspondence: Professor H Li, Ministry of Education, Key Laboratory for Liquid-Solid Structural Evolution and Processing of Materials, Shandong University, Jinan 250061, China.

E-mail: lihuilmy@hotmail.com

or Assistant Professor L Li, Faculty of Applied Science and Textiles, The Institute of Textiles and Clothing, The Hong Kong Polytechnic University, Hong Kong, China. E-mail: tclili@inet.polyu.edu.hk
}

Received 11 December 2012; revised 31 March 2013; accepted 6 May 2013 
into ferromagnetic metals. ${ }^{30,31}$ Sanvito and coworkers ${ }^{32}$ reported that a transverse electric field can direct the non-magnetic semiconducting single-layer armchair $\mathrm{MoS}_{2}$ nanoribbons to become conducting and magnetic, whereas bilayer $\mathrm{MoS}_{2}$ nanoribbons can be changed from indirect bandgap semiconductors to direct bandgap semiconductors. All of these findings provide new directions for reversibly changing the electron transport properties of nano-electronics, including spintronics and optotronics.

Graphene-like materials have been attracting increasing attention because they have significant advantages in low-dimensional scientific research and in nano-device applications. In 2012, Alejandro predicted the existence of boron phosphide (BP) nano-flakes, which exhibit intermediary electronic properties between those of graphene and $\mathrm{BN}$ systems. ${ }^{33}$ Zigzag BP nanoribbons ( $z$ BPNRs) and $z$ SiCNRs share the same crystal structure and may have very similar lattice constants. Moreover, their electronic properties should be different due to the different electronic structures of $\mathrm{C}, \mathrm{Si}, \mathrm{B}$ and $\mathrm{P}$. Therefore, these nanoribbons might be utilized to construct hybrid nano-structures with stable coherent interfaces and multifunctionality. Herein, we propose several types of simple nanostructures consisting of $z$ SiCNRs and $z$ BPNRs for constructing multi-functional nano-electronics, such as planar, ' $\mathrm{Y}$ '- and ' $\Delta$ 'shaped structures. We hypothesize that these nano-structures can exhibit FET characteristics, NDR effects and potential for spintronic applications. Furthermore, we anticipate that different from $z$ BNNRs, $z$ BPNRs can exhibit pronounced spin polarization properties under a transverse electric field.

\section{MATERIALS AND METHODS}

All calculations were performed within the first-principles density functional theory (DFT) and the non-equilibrium Green function frameworks using the Atomistix ToolKit software package. ${ }^{34,35}$ Local atomic numerical orbitals composed of double-zeta single polarized basis sets together with normconserving pseudo potentials were employed. Perdew-Burke-Ernzerhof generalized gradient approximations were used to describe the exchangecorrelation energy. The mesh cutoff for electrostatic potentials was $75 \mathrm{Ha}$.
A $1 \times 1 \times 50$ Monkhorst-Pack $k$-point sampling was employed to calculate the Brillouin zone integration. The electron temperature was set to $300 \mathrm{~K}$. To avoid interactions between periodic images, all structures were modeled in a supercell with vacuum layers of at least $15 \AA$ between neighboring cells. The geometries of the devices and of the $z$ BPNR crystal structures were optimized until the forces of the atoms were less than 0.05 and $0.01 \mathrm{eV}^{-1}$, respectively. The tolerance for energy convergence was $10^{-5} \mathrm{eV}$. The device current $I_{\mathrm{d}}$ was obtained using the Landauer-Büttiker equation ${ }^{34}$ :

$$
I=\frac{2 e}{h} \int_{-\infty}^{\infty} \mathrm{d} E\left(T(E, V)\left(f_{1}(E)-f_{2}(E)\right)\right),
$$

where $T(E, V)$ represents the quantum mechanical transmission probability for electrons, $f_{1,2}(E)$ denote the Fermi functions of the source and drain electrodes, and $e$ and $h$ are the electron charge and the Planck constant, respectively.

\section{RESULTS AND DISCUSSION}

Figure 1a shows the structure of the hexagonal BP sheet. Our calculated $\mathrm{B}-\mathrm{P}$ bond length was $1.858 \AA$, which is very close to the previously calculated $\mathrm{Si}-\mathrm{C}$ bond length $(1.787 \AA$ ) of the hexagonal $\mathrm{SiC}$ sheet, ${ }^{28}$ indicating the possibility of constructing hybrid BP-SiC structures with coherent interfaces. The band structure of the hexagonal BP sheet (Figure 1c) revealed that it is a direct bandgap semiconductor with its highest occupied valence band maximum (HOVBM) and lowest unoccupied conductance band minimum (LUCBM) both located at the $K$ point in the hexagonal Brillouin zone. The Bloch states of the HOVBM and the LUCBM are $\pi$ states localized at the $\mathrm{P}$ and $\mathrm{B}$ atoms, respectively (see the insets of Figure 1a). Moreover, it can be found that the bandgap $(0.87 \mathrm{eV})$ of the hexagonal BP sheet is very suitable for electronic applications.

Figure $1 \mathrm{~b}$ shows the structure of $z \mathrm{BPNRs}$ with hydrogen-passivated edges. These nanoribbons are classified by the number of zigzag BP chains $(N)$ along the $y$ axis and are denoted as NzBPNR. In contrast to $z$ BNNRs with indirect bandgaps, ${ }^{26} z$ BPNRs have direct bandgaps. Both the HOVBM and LUCBM are located at the $K_{M}$ point in the Brillouin zone (Figure 1d). As shown in Figure 1d, both the HOVB and the LUCB have a noticeable flat component from the $K_{M}$ point to
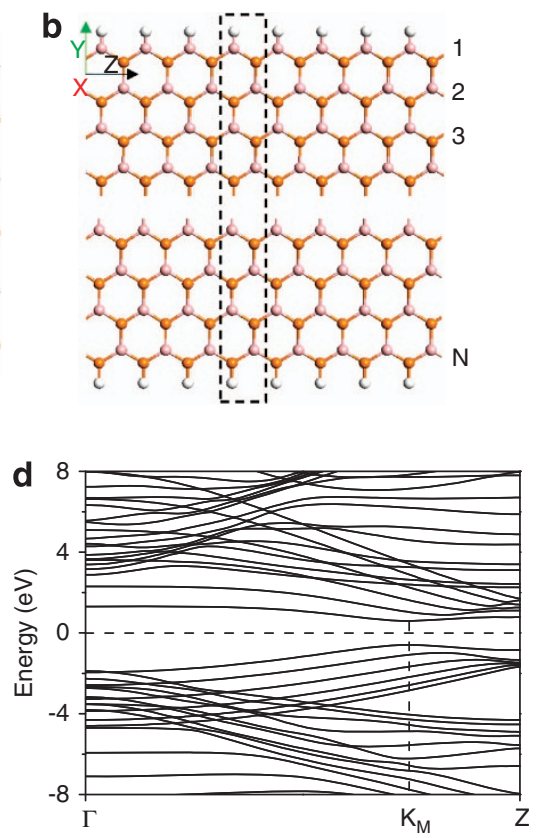

Figure 1 Structures of the hexagonal boron phosphide (BP) sheet (a) and the zigzag boron phosphide nanoribbon (zBPNRs) with edges passivated by hydrogen (b). The arrows and the dashed box denote the unit cell. (c), (d) are the band structures of the BP sheet and the 6zBPNR, respectively.
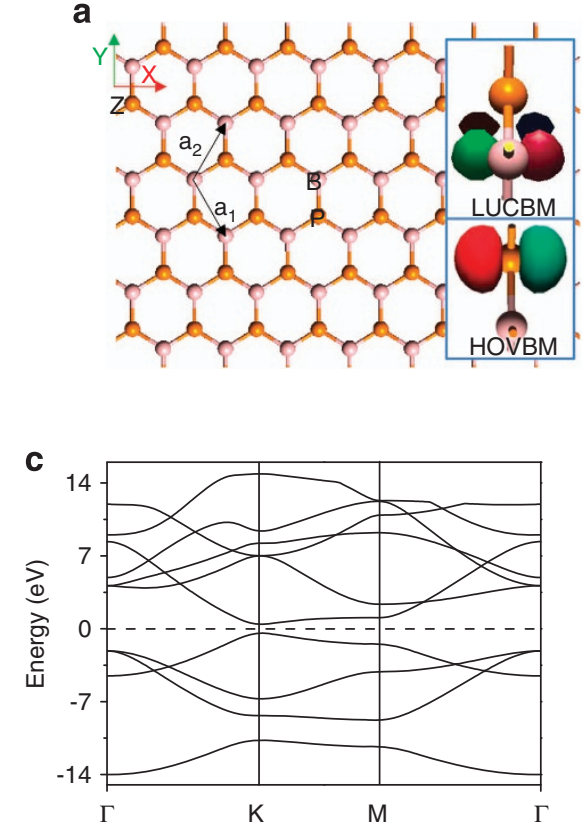
a
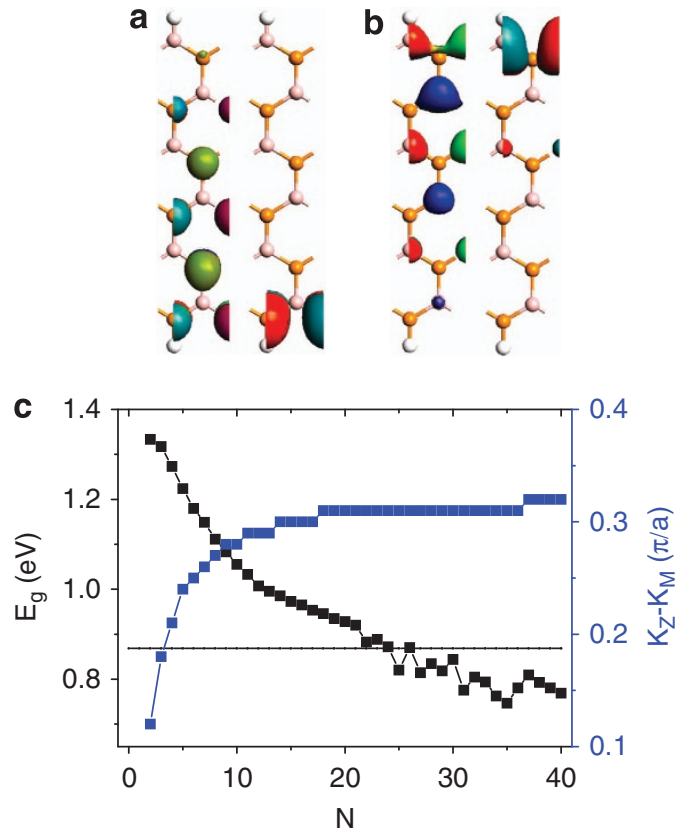

Figure 2 (a) The Bloch states at the highest occupied valence band maximum (HOVBM) (left panel) and the HOVBK $K_{Z}$ (right panel), and (b) the Bloch states at the lowest unoccupied conductance band minimum (LUCBM) (left panel) and the LUCBK $K_{Z}$ (right panel). The colors represent the phase. The isovalue is 0.1 . (c) The variation in the bandgap and the $K_{Z}-K_{M}$ of zigzag boron nitride nanoribbons (zBNNRs) as the ribbon width $N$ increases.

the $K_{Z}$ point, different from others. As shown in Figure 2a, the HOVBM of the $6 z \mathrm{BPNR}$ corresponds to the edge state localized at the $\mathrm{P}$ atoms decaying from the $\mathrm{P}$ to $\mathrm{B}$ edge (left panel), while the HOVB at the $K_{Z}$ point only corresponds to the $\mathrm{P}$ atoms at the $\mathrm{P}$ edge. Likewise, the LUCBM and the LUCB at the $K_{Z}$ point have the same features as the HOVBM and the HOVB at the $K_{Z}$ point (Figure $2 b$ ). Therefore, the bandgaps of $z$ BPNRs should be greatly affected by their edge states.

To understand the effects of the ribbon width $N$ on the bandgaps $\left(E_{\mathrm{g}}\right)$ of $z$ BPNRs, the relationship between $E_{\mathrm{g}}$ and $N$ is shown in Figure 2c. Notably, the $E_{\mathrm{g}}$ decreased monotonously as $N$ increased from 2 to 21 . Further increasing $N$ to 40 induced the $E_{\mathrm{g}}$ to decrease with fluctuations. Moreover, the $E_{\mathrm{g}}$ became lower than that of the hexagonal BP sheet if $N$ exceeded 24, which cannot be explained by the quantum confinement effect. In fact, these phenomena are attributed to two factors: one is the transfer of electrons between $\mathrm{B}$ and $\mathrm{P}$ atoms, and the other is the screening effect from the inner part of the ribbon. Owing to the different electronegativities of $\mathrm{B}$ and $\mathrm{P}$, the electron transfer from the $\mathrm{P}$ edge to the $\mathrm{B}$ edge of the $z \mathrm{BPNRs}$ results in the spontaneous formation of an electric field and the screening effect. As $N$ increased, fewer electrons were accumulated (depleted) at the B (P) edge, as reflected by the increase of the distance between the $K_{M}$ point and the $K_{Z}$ point (as shown in Figures $1 \mathrm{~d}$ and $2 \mathrm{c}$ ), which can be used to estimate the density of states of the $\mathrm{B}$ and $\mathrm{P}$ edges. Thus, the site energy at the $\mathrm{B}(\mathrm{P})$ edge decreased (increased), which caused the $E_{\mathrm{g}}$ to decrease. When the ribbon width was sufficiently large, the screening effect began to emerge and to compete with the electron transfer effect, leading to the bandgap fluctuation shown in Figure 2c. From the above discussion, it can be concluded that $z$ BPNRs may possess potential for applications in optotronics and logic electronics.
Previous studies have shown that the $6 z \mathrm{SiCNR}$ is a ferrimagnetic semiconductor with a negligible bandgap. In addition, the nonmagnetic state of the $6 z \mathrm{SiCNR}$ is also semiconducting with a very small bandgap. The energy difference between the non-magnetic and ferrimagnetic states of the $6 z \mathrm{SiCNR}$ is very small. ${ }^{29}$ This spinpolarized state would become unstable under external fields. ${ }^{36}$ Therefore, for practical purposes, a spin-unpolarized calculation was employed to investigate the electron transport properties of the planar hybrid $z$ SiC-BP-SiC nanoribbon devices. Figure $3 a$ presents a schematic of such a hybrid device. The dashed boxes denote the supercells of the source and drain electrodes. The scattering region, consisting of a hybrid $z \mathrm{SiC}-\mathrm{BP}-\mathrm{SiC}$ structure, was located between these two electrodes. A back-gate electrode (denoted by the gray box) can be added to this device to construct a FET. The current-voltage $\left(I_{\mathrm{d}}-V_{\mathrm{d}}\right)$ curves of this device (with no back gate) are shown in Figure $3 \mathrm{~b}$. Owing to the discrete energy levels of the scattering region, an obvious non-linear feature appeared. Moreover, a significant conductance gap appeared in the conductance curve, which resulted from the semiconducting feature of the $z \mathrm{BPNR}$. Interestingly, this twoprobe device exhibited significant NDR asymmetrically located at the $V_{\mathrm{d}}$ of -0.8 and $1 \mathrm{~V}$. This asymmetry was clearly induced by the asymmetric structure of the scattering region. Further analysis revealed that the NDR arises from the change in the SiC-BP coupling under various $V_{\mathrm{d}}$.

To understand the mechanism underlying the electron transport properties of this two-probe device, its transmission spectrum and local density of states (LDOS) ${ }^{37}$ at different $V_{\mathrm{d}}$ were calculated (Figure 4). The LDOS was averaged in the $x-y$ plane and colored with red and blue, denoting large and small density areas, respectively. The reference energy was set as the Fermi energy $\left(E_{\mathrm{f}}\right)$ of the electrodes at the $V_{\mathrm{d}}$ of $0 \mathrm{~V}$. When the $V_{\mathrm{d}}$ was $0 \mathrm{~V}$, the corresponding transmission spectrum displayed a clear transmission energy gap of $\sim 0.7 \mathrm{eV}$ around the $E_{\mathrm{f}}$ (Figure $4 \mathrm{a}$ ), which originated from the semiconducting behavior of the $z$ BPNR, suggesting that such hybrid devices have potential applications in FETs. ${ }^{38}$ In addition, there was a very small transmission peak at the $E_{\mathrm{f}}$, as shown in the inset in Figure $4 \mathrm{a}$, which has a crucial role in the abovementioned NDR. This small transmission peak was induced by the quantum tunneling effect of electrons between the two $z$ SiCNRs located at the two sides of the $z$ BPNR. As indicated from the LDOS in Figure $4 \mathrm{~d}$, the DOS at the $E_{\mathrm{f}}$ mainly distributed at the $z \mathrm{SiCNR}$ and the SiC-BP interfaces, and there was no electron state at the $z \mathrm{BPNR}$ region. Therefore, electrons at the $E_{\mathrm{f}}$ were only able to penetrate the scattering region via the tunneling effect. As the $V_{\mathrm{d}}$ decreased from 0 to $-0.8 \mathrm{~V}$, the chemical potential of the left (right) electrode $\mu_{1}\left(\mu_{2}\right)$ decreased (elevated) by $0.4 \mathrm{eV}$ (as shown in Figure 4b). Meanwhile, the transmission of electrons via the tunneling effect at $\sim \pm 0.4 \mathrm{eV}$ was enhanced, as reflected by the LDOS in Figure 4e. Such changes of the transmission spectrum in the $\mu_{1}-\mu_{2}$ energy window led to an exponential increase of $I_{\mathrm{d}}$ (see the inset in Figure $3 \mathrm{~b}$ ). When $V_{\mathrm{d}}$ was further decreased, the electron transmission mechanism began to transition from pure tunneling to resonant tunneling through the tails of the highest occupied molecular orbital and the lowest unoccupied molecular orbital of the $z$ BPNR; this transition resulted in a decrease of the electron transmission in the $\mu_{1}-\mu_{2}$ energy window and thus in the appearance of NDR (Figures $4 \mathrm{c}$ and f). According to the above analysis, we can hypothesize that it is possible to control the tunneling effect and subsequently the NDR effect through changing the length of the $z$ BPNR. This hypothesis was confirmed by Figure $3 \mathrm{c}$, which shows the $I_{\mathrm{d}}-V_{\mathrm{d}}$ curve of a $z \mathrm{SiC}$-BP-SiC two-probe device with a $z$ BPNR consisting of six periodic units. Clearly, due to the short $z \mathrm{BPNR}$, 


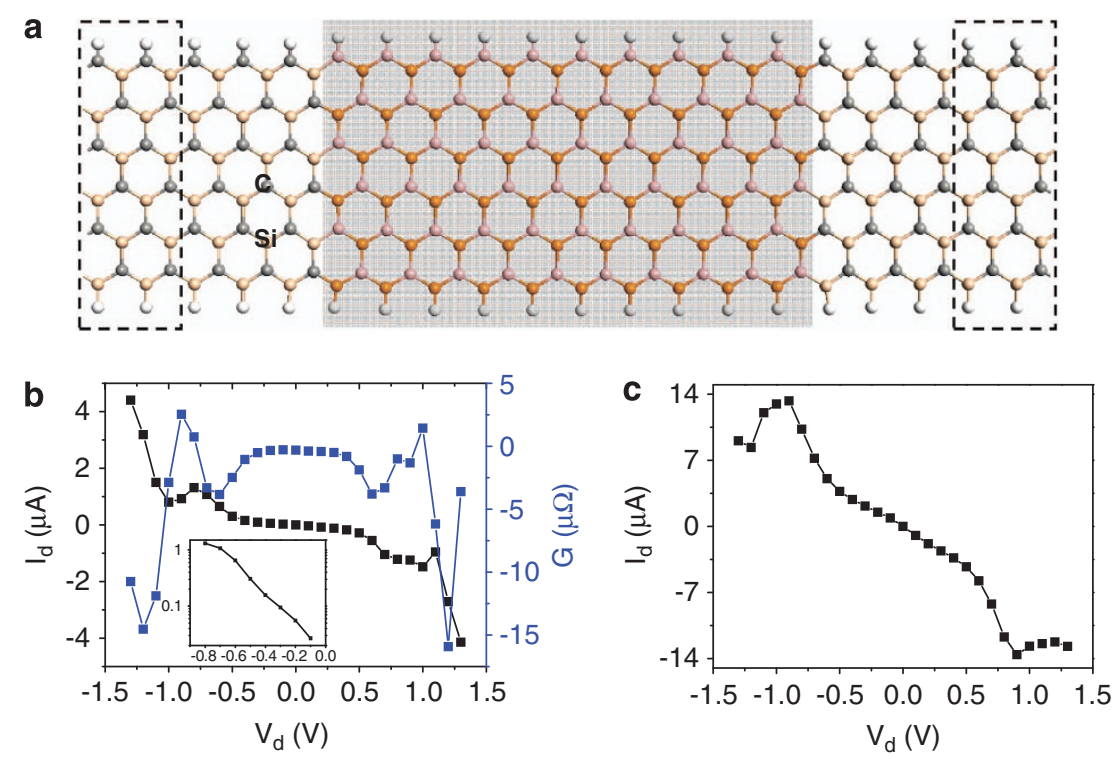

Figure 3 (a) Schematic of a $z \mathrm{SiC}-\mathrm{BP}-\mathrm{SiC}$ nanoribbon transistor. (b) $I_{\mathrm{d}}-V_{\mathrm{d}}$ and conductance curves of the $z \mathrm{SiC}-\mathrm{BP}-\mathrm{SiC}$ two-probe device. The inset shows the $\mathrm{Ig} I_{\mathrm{d}}-V_{\mathrm{d}}$ curve. (c) $I_{\mathrm{d}}-V_{\mathrm{d}}$ curve of a $z \mathrm{SiC}-\mathrm{BP}-\mathrm{SiC}$ two-probe device with a shorter zigzag boron phosphide nanoribbon (zBPNR) than that shown in (b).

a
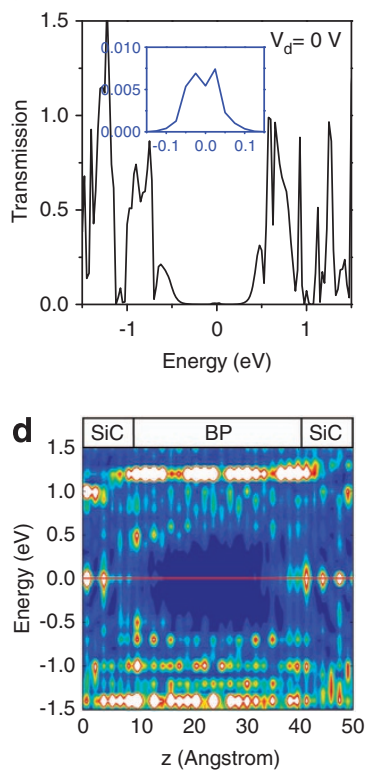

b
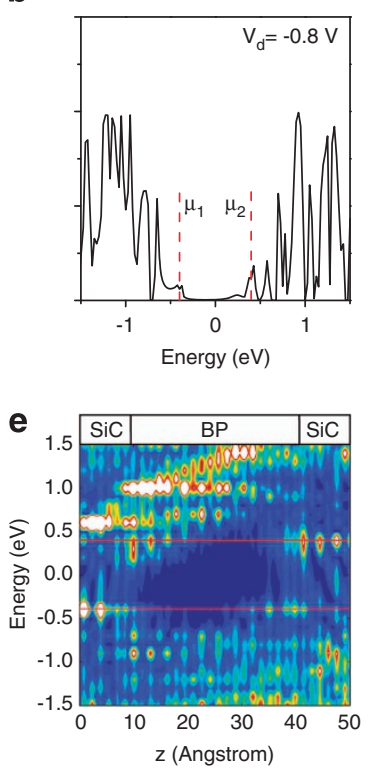

C
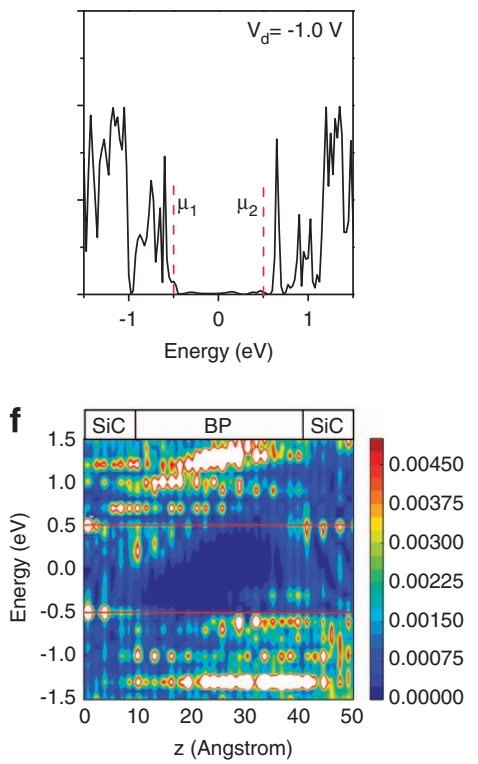

Figure 4 Transmission spectra (a), (b) and (c), and local density of states (d), (e) and (f) of the two-probe device shown in Figure $3 a$ at $V_{d}$ of 0 , -0.8 and $-1.0 \mathrm{~V}$, respectively.

the tunneling current was considerably stronger than the one discussed above. More importantly, this two-probe device also exhibited significant NDR, which was asymmetrically located at $\sim \pm 0.9 \mathrm{~V}$, indicating that the NDR effect of such $z \mathrm{SiC}-\mathrm{BP}-\mathrm{SiC}$ devices is controllable. In fact, a similar NDR has been observed in $\mathrm{C}_{60}$-based structures, but those structures are much more complicated than the ones described here. ${ }^{39}$ In addition, those structures may become unstable due to sophisticated interface coupling.

The electron transport properties of a diode-like structure constructed using the $z \mathrm{BP}-\mathrm{SiC}$ nanoribbon were also studied. Figure $5 \mathrm{a}$ shows the $I_{\mathrm{d}}-V_{\mathrm{d}}$ curve of a $5 z \mathrm{BP}-\mathrm{SiC}$ two-probe device. Owing to the semiconducting feature of the $z$ BPNRs, there was a clear conductance gap of $\sim 1 \mathrm{~V}$ in the $I_{\mathrm{d}}-V_{\mathrm{d}}$ curve of the $5 z \mathrm{BP}-\mathrm{SiC}$ two-probe device. Moreover, this two-probe device exhibited a slight NDR when the applied bias was $\sim \pm 1 \mathrm{~V}$. As inferred from the transmission spectra and the LDOS shown in Figure 6, such NDR characteristics also arise from the change in coupling between the $z$ BPNR and the $z$ SiCNR under different applied biases. Comparing Figures $6 \mathrm{~d}$ with e reveals that applying a $-0.9 \mathrm{~V}$ bias lowers (elevates) the chemical potential of the left (right) electrode. Consequently, electrons flow through the lowest unoccupied molecular orbital of the $z$ BPNR and through the highest occupied molecular orbital and the lowest unoccupied molecular orbital of the $z \mathrm{SiCNR}$ in the scattering region (as shown in Figure 6e), leading to the increase of the current. However, when 

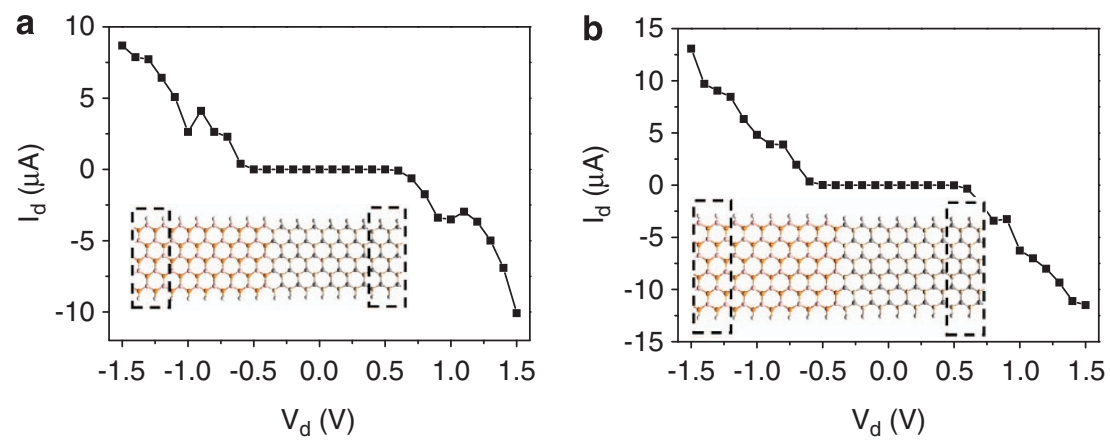

Figure 5 (a) $I_{\mathrm{d}}-V_{\mathrm{d}}$ curve of the $5 z \mathrm{BP}-\mathrm{SiC}$ two-probe device, and (b) $I_{\mathrm{d}}-V_{\mathrm{d}}$ curve of the $6 z \mathrm{BP}-\mathrm{SiC}$ two-probe device. The insets are the structures of these devices.
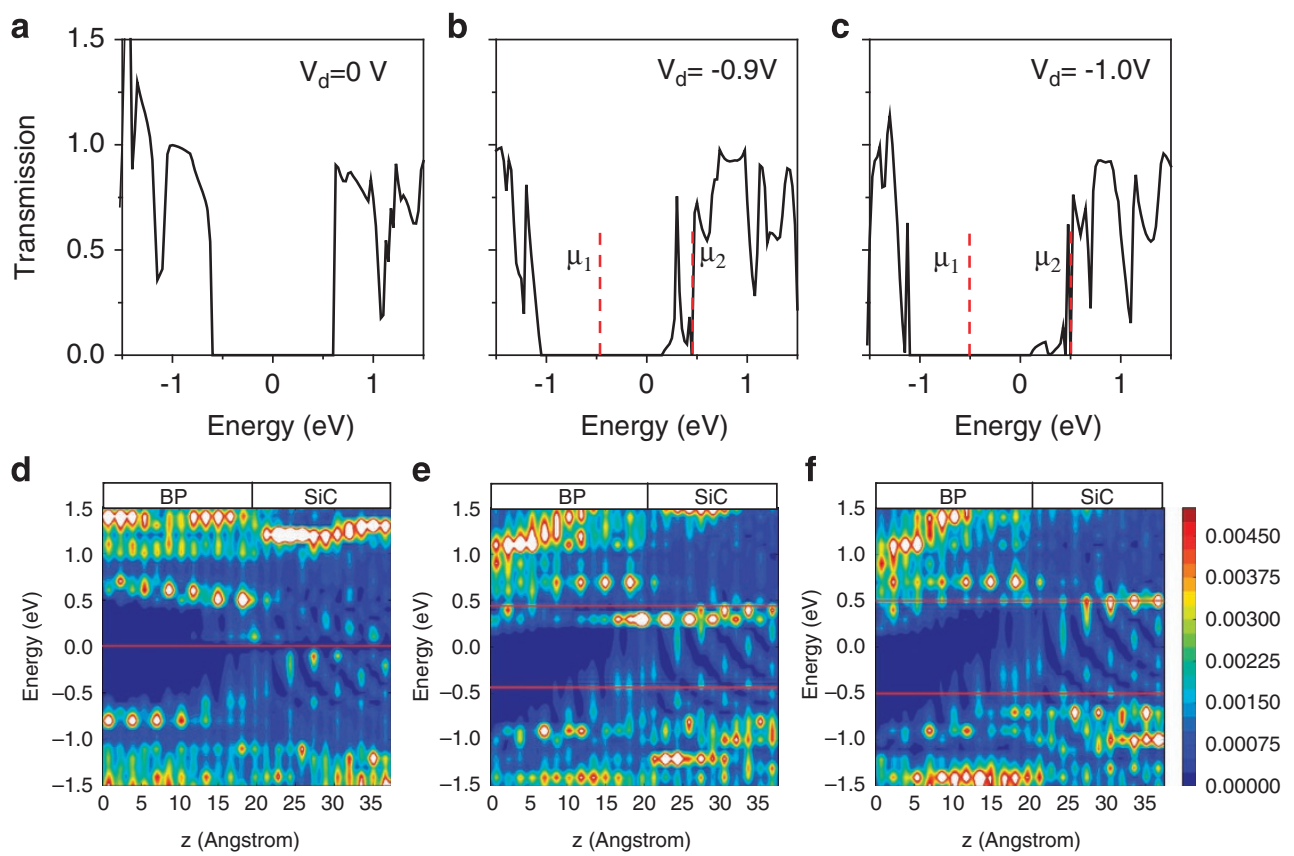

Figure 6 Transmission spectra (a), (b) and (c), and local density of states (d), (e) and (f) of the two-probe device shown in Figure $5 a$ at $V_{d}$ of $0,-0.9$ and $-1.0 \mathrm{~V}$, respectively.

the bias was decreased to $-1.0 \mathrm{~V}$, the contribution of the highest occupied molecular orbital of the $z$ SiCNR to the current was significantly abated, which caused the current to decrease (Figures $6 \mathrm{c}$ and $\mathrm{f}$ ). The $I_{\mathrm{d}}-V_{\mathrm{d}}$ curve of the $6 z \mathrm{BP}-\mathrm{SiC}$ two-probe device (shown in Figure $5 b$ ) demonstrates that this type of NDR would weaken or disappear as the width of the ribbon increases.

To investigate the importance of the $z$ BPNRs in the two types of devices mentioned above, the electron transport properties of folded $4 z \mathrm{SiCNRs}$ were investigated. As shown in the insets in Figure 7, the electrodes of these devices were composed of ideal $4 z \mathrm{SiCNR}$ and the scattering regions were composed of folded $4 z$ SiCNRs, with the overlapping area decreasing from device a to d. The transmission spectra presented in Figure 7 indicate that none of these devices exhibited electron transmission energy gaps near the Fermi energy. Such results are reasonable because the non-magnetic $4 z \operatorname{SiCNR}$ is a narrow gap semiconductor. Therefore, it is very difficult to construct nano-electronics using only planar $z$ SiCNR structures.

Apart from the planar hybrid structures, $z$ SiCNRs and $z$ BPNRs easily form hybrid structures with other shapes. For instance, Figure 8 presents schematic illustrations of two types of ' $\mathrm{Y}$ '-shaped tri-wing hybrid structures composed of $z \mathrm{SiCNRs}$ and $z \mathrm{BPNRs}$, denoted tri-SiC and tri-SiC-BP, respectively. The tri-SiC consisted of three $4 z \mathrm{SiCNRs}$ with two Si edges and one $\mathrm{C}$ edge connected to each other through $s p^{3}$ hybridization, whereas the tri-SiC-BP consisted of two $4 z$ SiCNRs and one $4 z \mathrm{BPNR}$ with two $\mathrm{Si}$ edges connected to one $\mathrm{P}$ edge. In addition, the $\mathrm{P}$ edge of the $4 z \mathrm{BPNR}$ in the stable tri-SiC-BP exhibited a very slight distortion. According to a previous study on the tri-wing GNR, ${ }^{40}$ these hybrid structures should be considerably stable against external disturbances. More importantly, due to the hybridization among the three wings, these ' $\mathrm{Y}$ '-shaped hetero-structures may exhibit intriguing electronic properties.

Figure 9 shows the electronic structure of the tri-SiC structure. As indicated from the total density of states (TDOS) (as shown in Figure 9e), together with the normalized spin density (Figure 9a), defined as $\left(\rho_{\text {up }}-\rho_{\text {down }}\right) /\left(\rho_{\text {up }}+\rho_{\text {down }}\right)$, where $\rho$ is the electron density, the tri-SiC is a ferrimagnetic metal with its ferrimagnetic electrons localized at the $\mathrm{H}$-passivated edges of each wing. In addition, wings 1 and 2 of the tri-SiC are ferromagnetically coupled, but they are anti-ferromagnetically coupled with wing 3 . This result can be further confirmed by the density of states projected on each 

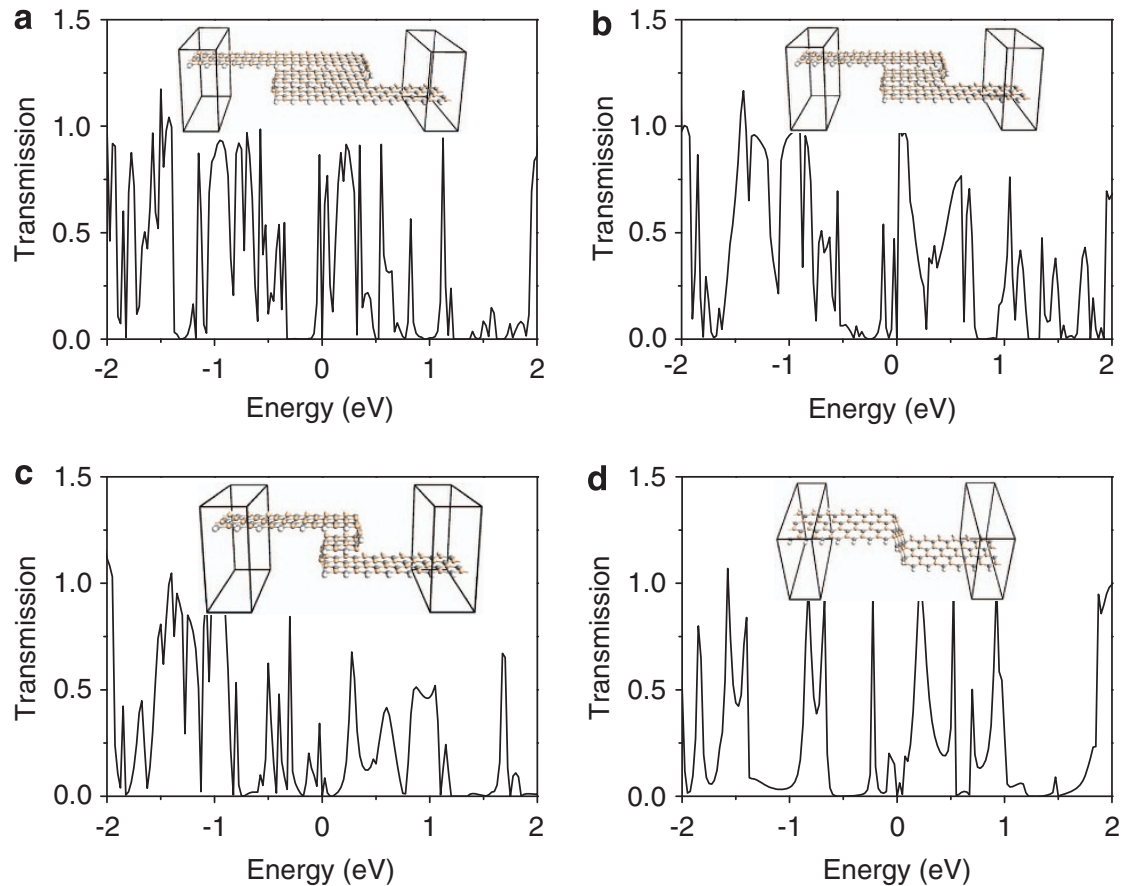

Figure 7 Transmission spectra of four folded $4 z$ SiCNR (silicon carbide nanoribbon) two-probe devices. The insets illustrate the corresponding structures of these devices.
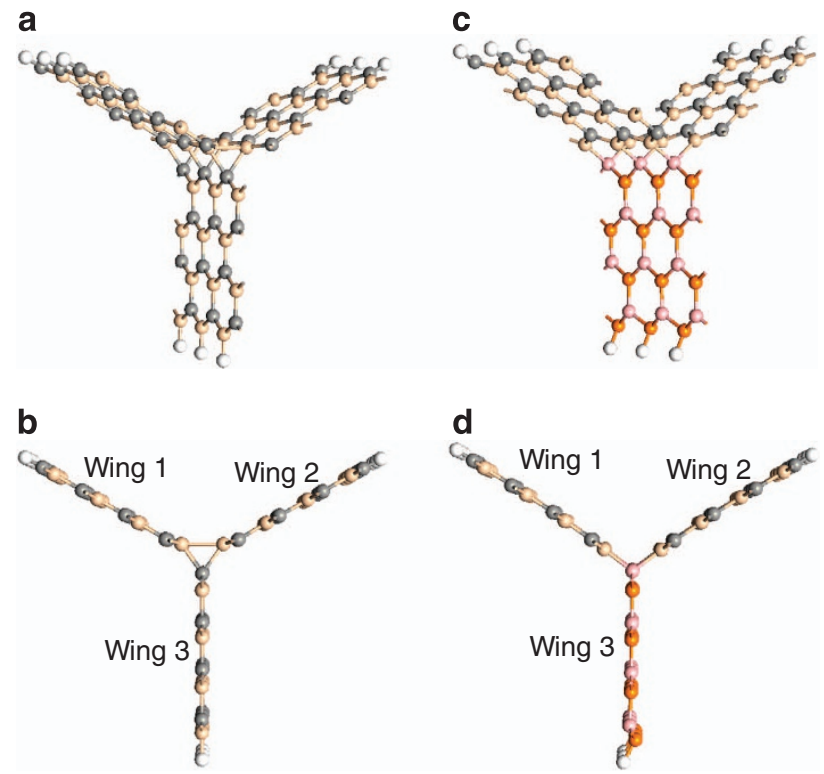

Figure 8 Structures of the tri-SiC (a, b) and the tri-SiC-BP (c, d) with three unit cells.

wing (PDOS) of the tri-SiC (Figure 9e). Therefore, the total magnetic moment of the tri-SiC is only $0.171 \mu_{\mathrm{B}}$ per unit cell. It is expected that if the three wings are coupled at the $\mathrm{Si}$ or $\mathrm{C}$ edges, the total magnetic moment per unit cell would be tripled. Interestingly, the central region of the tri-SiC was almost non-magnetic, which is in contrast to the tri-wing GNR. ${ }^{40}$ In the band structure (as shown in Figure 9d), there were two flat bands at $\sim 0.1 \mathrm{eV}$ above the Fermi energy level for spin-up and spin-down species. The corresponding Bloch states at the
$\Gamma$ point (as shown in Figures $9 \mathrm{~b}$ and $\mathrm{c}$ ) indicated that only the spinup flat band is induced by the hybridization of three wings and that the spin-down flat band actually resulted from the $p$ states of the $\mathrm{Si}$ atoms at the Si edge of wing 3. This is an additional property that differs from the tri-wing GNR. ${ }^{40}$

Figure 10 shows that the tri-SiC-BP structure was also ferrimagnetic and metallic. However, the tri-SiC-BP structure exhibited rather different electronic properties compared with the tri-SiC structure. First, in the tri-SiC-BP structure, only the two $z \mathrm{SiCNR}$ wings exhibited ferrimagnetic properties, with the corresponding electrons localized at the H-passivated edges, whereas the $z \mathrm{BPNR}$ wing was almost non-magnetic (as shown in Figures 10a and e), leading to a $0.411-\mu_{\mathrm{B}}$ total magnetic moment per unit cell, which is expectedly comparable with the double value of the tri-SiC. Moreover, although two flat bands also appeared at $\sim 0.1 \mathrm{eV}$ above the Fermi energy level for the spin-up and spin-down species (see Figure 10d), both of these bands were primarily composed of Si-B $\sigma$ bonds and the $p$ states of the $\mathrm{P}$ atoms localized at the central region of the tri-SiC-BP (as shown in Figures 10b and c).

As both the tri-SiC and the tri-SiC-BP exhibit peculiar electronic properties, exploring their possible applications in electronic devices is of importance. Figures 11a and b show the electron transmission spectra of the tri-SiC and tri-SiC-BP two-probe devices, respectively. It can be observed that these transmission spectra display a step-like shape because there are no electron scattering sources. These devices clearly exhibited pronounced spin polarization characteristics near the Fermi level. For the tri-SiC two-probe device, its spin polarization ratio around the Fermi level was $80 \%$, indicating that $80 \%$ of the electrons are spin-down under a small bias. Further analysis revealed that this ratio was even higher around the $\mathrm{H}$-passivated edges. The spin polarization ratio around the Fermi level of the tri-SiC-BP twoprobe device was $75 \%$. Similarly, this ratio became much higher around the two $\mathrm{H}$-passivated $\mathrm{C}$ edges. In addition, due to the almost 

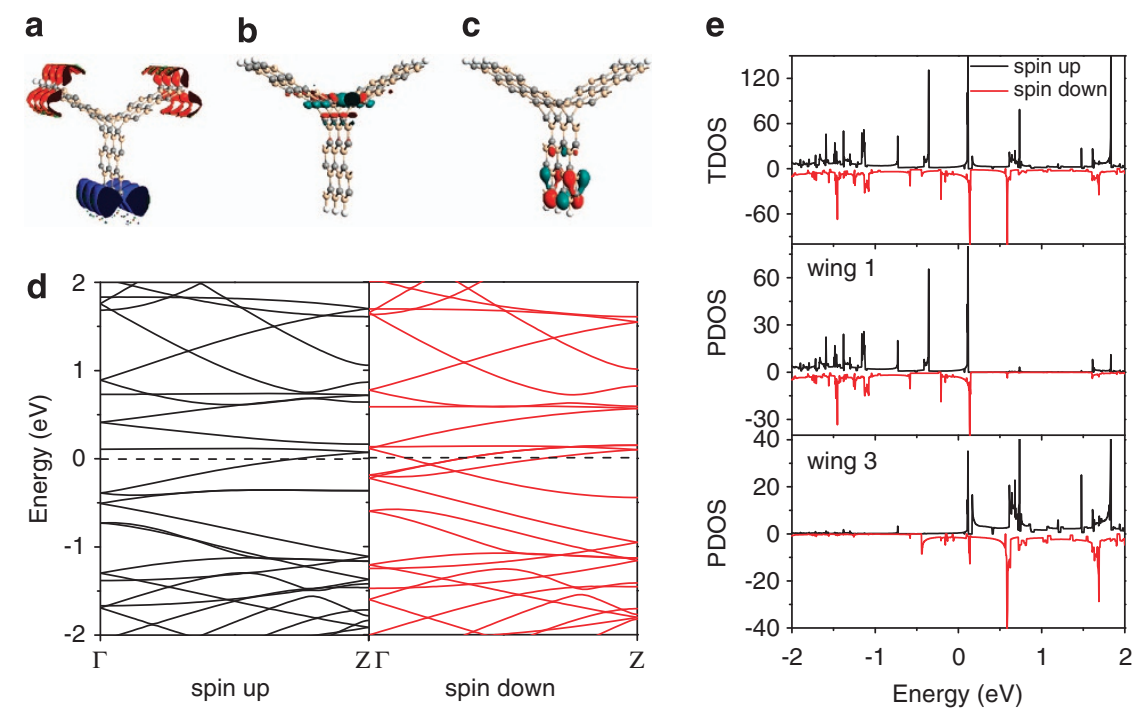

Figure 9 (a) Normalized electron spin density difference, where red and blue represent positive and negative, respectively; (b, c) the Bloch states at the $\Gamma$ point of the spin-up and spin-down flat bands, respectively; (d) the band structure of the tri-wing SiC; and (e) the TDOS and PDOS of the tri-wing SiC.
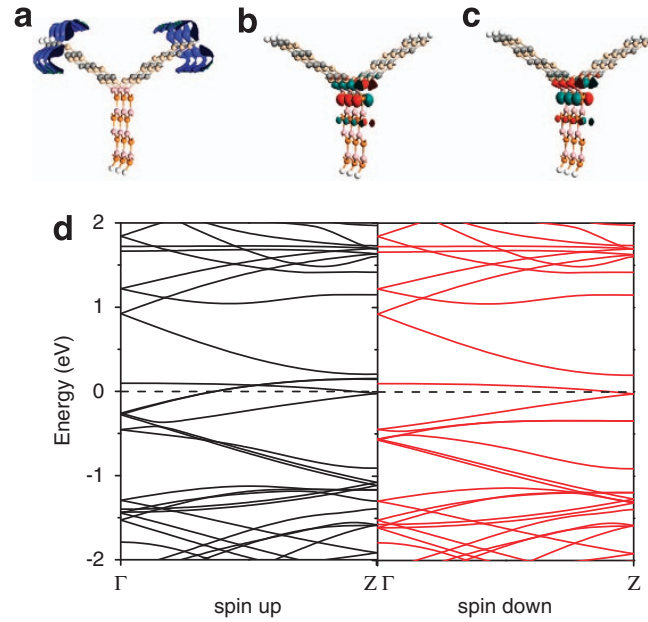

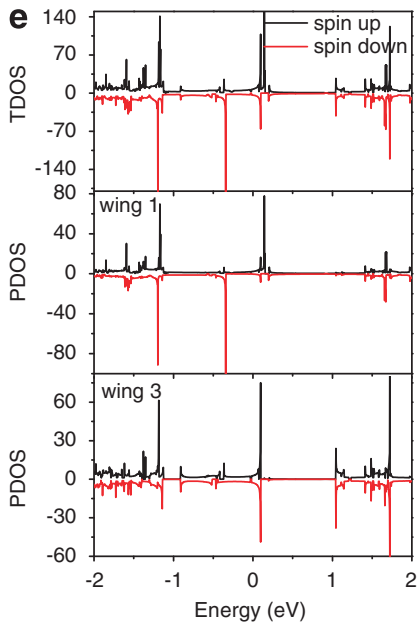

Figure 10 (a) Normalized electron spin density difference; (b, c) the Bloch states of the spin-up and spin-down flat bands at the $\Gamma$ point, respectively; (d) the band structure of the tri-wing SiC-BP; and (e) the TDOS and PDOS of the tri-wing SiC-BP.
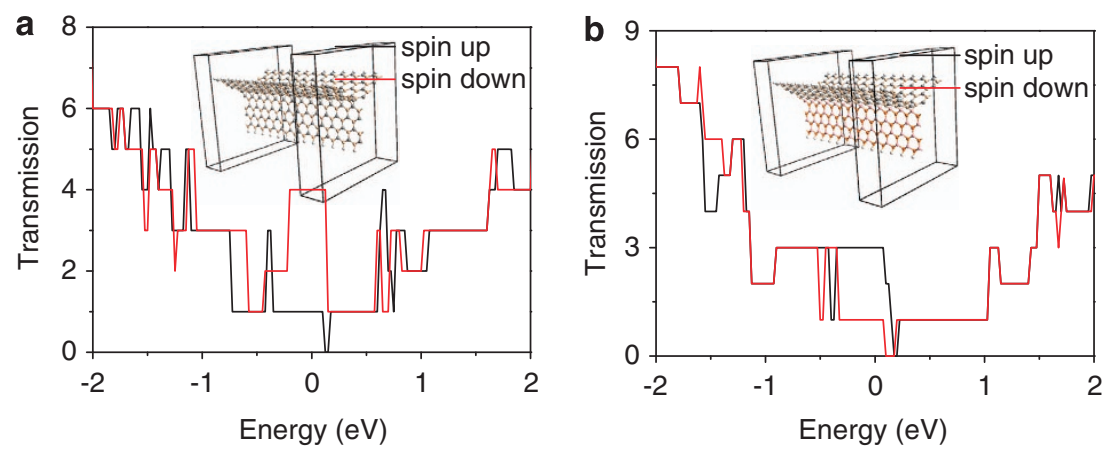

Figure 11 (a) Transmission spectra of the tri-SiC two-probe device, and (b) transmission spectra of the tri-SiC-BP two-probe device. The insets illustrate the structure of these two devices, with their electrodes denoted by black boxes.

non-magnetic feature behavior of the $z$ BPNR wing, electrons that were transported through this region exhibited little spin polarization.

It is well known that nanoribbons can be zipped into nanotubes. As demonstrated above, the electronic properties of $z$ SiCNR- and $z$ BPNR- based structures are critically dependent on their edge states. If these nanoribbons are zipped into nanotubes, the edge states should disappear and consequently lead to different properties. Figures $12 \mathrm{a}$ and d show the structures of an armchair $\mathrm{SiC}$ nanotube (SiCNT) and an armchair 

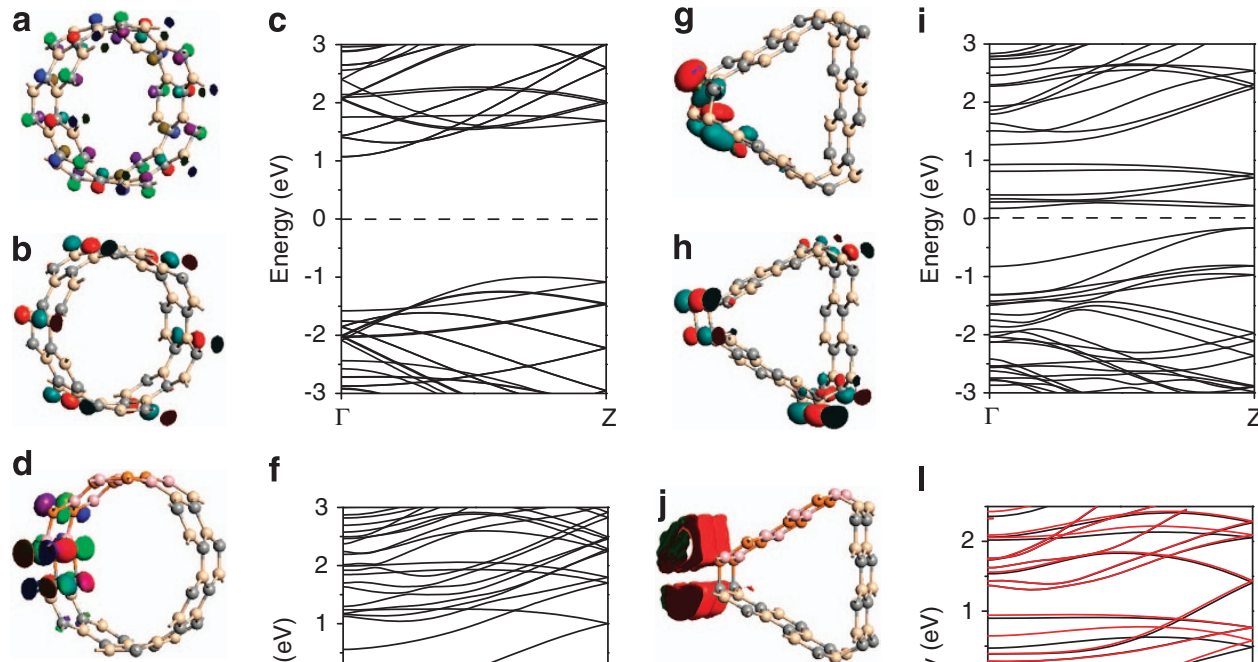

f
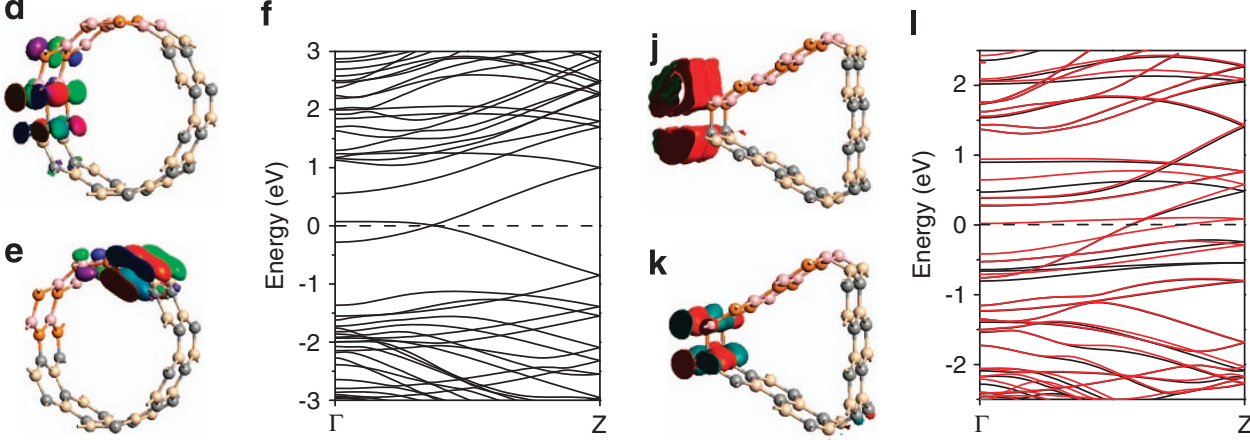

Figure 12 The highest occupied valence band maximum (HOVBM) (a), the lowest unoccupied conductance band minimum (LUCBM) (b) and band structure (c) of the SiC nanotube (SiCNT); the HOVBM (d), LUCBM (e) and band structure (f) of the SiC-BP nanotube (SiC-BPNT); the HOVBM (g), LUCBM (h) and band structure (i) of the ' $\triangle$ '-shaped SiC tube; the normalized spin density (j), Bloch state of the spin-down flat band at the $\Gamma$ point near the Fermi energy level (k) and band structure (I) of the ' $\Delta$ '-shaped SiC-BP tube (black and red lines denote spin up and spin down, respectively).

SiC-BP nanotube (SiC-BPNT), respectively. The SiCNT was composed of a $12 z \mathrm{SiCNR}$, whereas the SiC-BPNT was constructed from an $8 z \operatorname{SiNR}$ and a $4 z \mathrm{BPNR}$. As indicated from the band structure (as shown in Figure 12c) of the SiCNT, this nanotube was a nonmagnetic indirect bandgap $\left(E_{\mathrm{g}} \sim 2.0 \mathrm{eV}\right)$ semiconductor with its HOVBM (LUCBM) distributed at the $\mathrm{C}(\mathrm{Si})$ atoms (as shown in Figures $12 \mathrm{a}$ and $\mathrm{b}$ ), in contrast to the $z$ SiCNRs. ${ }^{28,29}$ Interestingly, substituting one-third of the SiCNT with a $4 z$ BPNR led to metallic behavior and two crossing bands near the Fermi energy level of the SiC-BPNT (Figure 12f). Figures 12d and e reveal that these two bands are primarily composed of the SiC-BP boundary states. Therefore, hybrid SiC-BP structures could be used to tune the properties of SiCNTs. Figures $12 \mathrm{~g}$ and $\mathrm{j}$ show the structures of the ' $\Delta$ '-shaped $\mathrm{SiC}$ and SiC-BP tubes, which have equal perimeters with the SiCNT and SiC-BPNT, respectively. Notably, the ' $\Delta$ '-shaped SiC tube exhibited a much smaller bandgap $(0.33 \mathrm{eV}$, shown in Figure 12i) than the SiCNT. The HOVBM of the ' $\Delta$ '-shaped SiC tube distributed at the $\mathrm{C}$ atoms near one vertex of the triangle (as shown in Figure 12g), and the LUCBM was localized at the $\mathrm{C}$ and $\mathrm{Si}$ atoms at three vertices (as shown in Figure 12h). Unlike the ' $\Delta$ '-shaped SiC tube, the ' $\Delta$ '-shaped SiC-BP tube was ferrimagnetically metallic with a magnetic moment of $0.569 \mu_{\mathrm{B}}$ per unit cell. As implied from the normalized spin density (as shown in Figure 12j), the spin polarization primarily occurred at the C-P vertex of the triangle. In addition, in the band structure (as shown in Figure 12l), there was a spin-down flat band near the Fermi energy. The corresponding Bloch state at the $\Gamma$ point indicated that this flat band was induced by the C-P boundary states (as shown in Figure 12k). The PDOS of the C-P boundary (not shown) indicated that most of the electrons near the Fermi energy level (from $E_{\mathrm{f}}-0.3$ to $E_{\mathrm{f}}+0.3 \mathrm{eV}$ ) in this region are spin down. Therefore, such hybrid SiC-BPNTs may have potential applications in spintronics, whereas pure $\mathrm{SiC}$ nanotubes can be used in logics.
Son et al. ${ }^{25}$ found that transverse electric fields can cause $z$ GNRs to transition into half-metals because of the electrostatic potential difference and the anti-ferromagnetic coupling between the two edges. $z \mathrm{BPNRs}$ have the same crystal structure as $z \mathrm{GNRs}$; therefore, determining whether $z$ BPNRs also exhibit similar properties as $z$ GNRs is of interest. As shown in Figures 13a, a transverse electric field was applied to the $8 z \mathrm{BPNR}$ by using a periodic open-circuit split-gate perpendicular to the ribbon axis in the unit cell. ${ }^{25}$ Figure $13 \mathrm{~b}$ shows the variation in the bandgap of the $8 z$ BPNR as a function of the field strength. (To take free-electron-like states above the Fermi level into consideration, ghost orbitals are employed in this part of calculation.) Apparently, owing to the broken symmetry of the $z$ BPNRs, the changes in the bandgap of the $8 z \mathrm{BPNR}$ differed in different field directions. For the positive direction, the $E_{\mathrm{g}}$ decreased monotonously and non-linearly as the field strength increased from 0 to $1.67 \mathrm{eV} \mathrm{nm}^{-1}$. Notably, the spin-up and spin-down bandgaps began to split when the field strength exceeded $1.67 \mathrm{eV} \mathrm{nm}^{-1}$, indicating that the $8 z \mathrm{BPNR}$ transitioned from a non-magnetic semiconductor to a half-metal. Under the negative electric field, the $E_{\mathrm{g}}$ increased with the field strength, whereas in the presence of a strong field, it first decreased quadratically and then linearly. Such changes of the bandgap of the 8zBPNR under the transverse electric field reflect the characteristics of both $z$ GNRs and $z$ BNNRs. ${ }^{25-27}$

To elucidate the novel phenomena described above, the change in the electronic structure of the $8 z \mathrm{BPNR}$ under various field strengths was investigated. Figure $13 \mathrm{c}$ shows the change in the Mulliken population of the $\mathrm{B}(\mathrm{P})$ atoms at the $\mathrm{B}(\mathrm{P})$ edge. Clearly, as the field strength increased, the Mulliken population of the $\mathrm{B}(\mathrm{P})$ atoms exhibited a significant increase (decrease), suggesting that the change in the electronic structure of the 8zBPNR under the positive transverse electric field was mediated by the transfer of electrons between $\mathrm{P}$ and $\mathrm{B}$ edge atoms. When the field strength was increased 

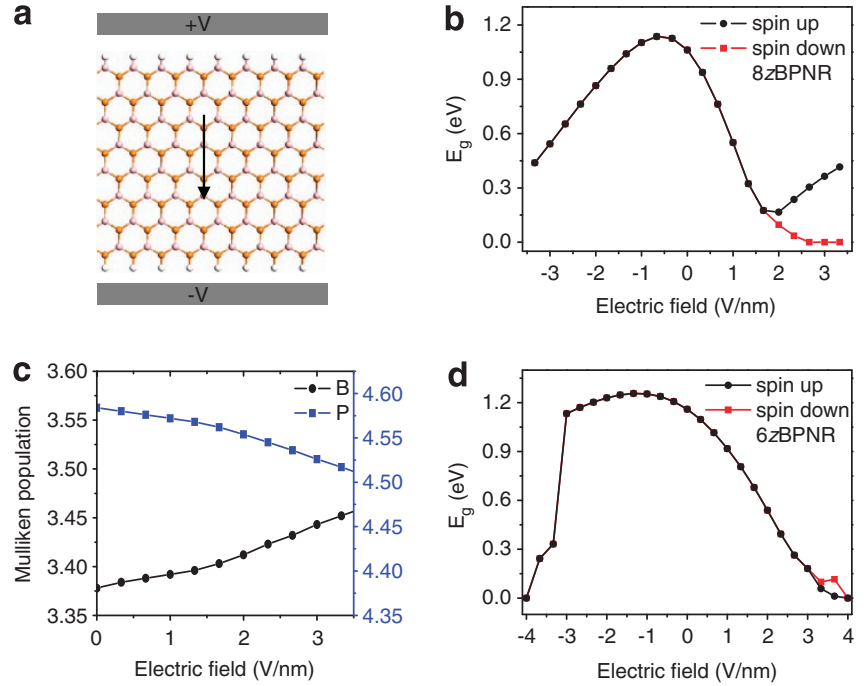

Figure 13 The schematic (a), bandgap variation (b) and Mulliken population variation of the edge $\mathrm{B}$ and $\mathrm{P}$ atoms (c) of the 8zBPNR (zigzag boron phosphide nanoribbon) under the transverse electric field, (d) the bandgap variation of the $6 z$ BPNR under the transverse electric field.

from 0 to $1.67 \mathrm{~V} \mathrm{~nm}^{-1}$, the electrostatic potential at the $\mathrm{P}$ (B) edge of the ribbon increased (lowered), resulting in the depletion (accumulation) of electrons at the $\mathrm{P}$ (B) edge. The change in the electrostatic potential at the ribbon edges decreased the bandgap, whereas the enhanced transfer of electrons from the $\mathrm{P}$ to the $\mathrm{B}$ atoms strengthened the spontaneously formed electric field and thus increased the bandgap. The combined result of the two co-existing factors led to the decrease of the bandgap. Additionally, as shown in Figure 15a, the $8 z$ BPNR changed from a non-magnetic direct bandgap semiconductor to a non-magnetic indirect bandgap semiconductor, with its HOVBM (LUCBM) located at the $K_{M}\left(K_{Z}\right)$ point localized at the $\mathrm{P}(\mathrm{B})$ atoms of the $\mathrm{P}(\mathrm{B})$ edge. When the strength of the transverse electric field was further increased $\left(>1.67 \mathrm{~V} \mathrm{~nm}^{-1}\right)$, the $8 z$ BPNR began to change to a ferrimagnetic semiconductor and eventually became halfmetallic. The spin-up HOVBM (LUCBM) of the $8 z \mathrm{BPNR}$ remained at the $\mathrm{P}(\mathrm{B})$ atoms of the $\mathrm{P}(\mathrm{B})$ edge, whereas the spin-down HOVBM (LUCBM) gradually distributed at the $\mathrm{B}(\mathrm{P})$ atoms of the $\mathrm{B}(\mathrm{P})$ edge (as shown in Figure 15b). Figure 14 indicates that as the field strength increased, the energy of the spin-up (down) $p$ electrons of the $\mathrm{P}$ atoms decreased (increased). In contrast, the energy of the spin-up (down) $p$ electrons of the B atoms increased (decreased). Consequently, when the field strength was $2.67 \mathrm{~V} \mathrm{~nm}^{-1}$, the energy of the spin-down $p$ electrons of the $\mathrm{P}$ atoms began to exceed that of the $\mathrm{B}$ atoms, making the spin-down HOVBM (LUCBM) of the 8zBPNR localize at the $\mathrm{B}(\mathrm{P})$ atoms. This bandgap splitting differs from that of antiferromagnetic $z \mathrm{GNRs}$, which originates from the electrostatic potential difference between two edges and is purely induced by the transverse electric field. ${ }^{25}$

Under the negative transverse electric field, the electrostatic potentials at the $\mathrm{B}$ and $\mathrm{P}$ edges of the $8 z \mathrm{BPNR}$ were increased and decreased, respectively, suppressing the transfer of electrons from the $\mathrm{P}$ to the $\mathrm{B}$ atoms. As the field strength increased, the Bloch state of the HOVBM (LUCBM) localized at the P (B) edge of the $8 z$ BPNR tended to move to the opposite site. As illustrated in Figure 15c, the Bloch state of the HOVBM (LUCBM) distributed at the $\mathrm{P}$ (B) atoms throughout the ribbon when the field strength was $-0.67 \mathrm{~V} \mathrm{~nm}^{-1}$.

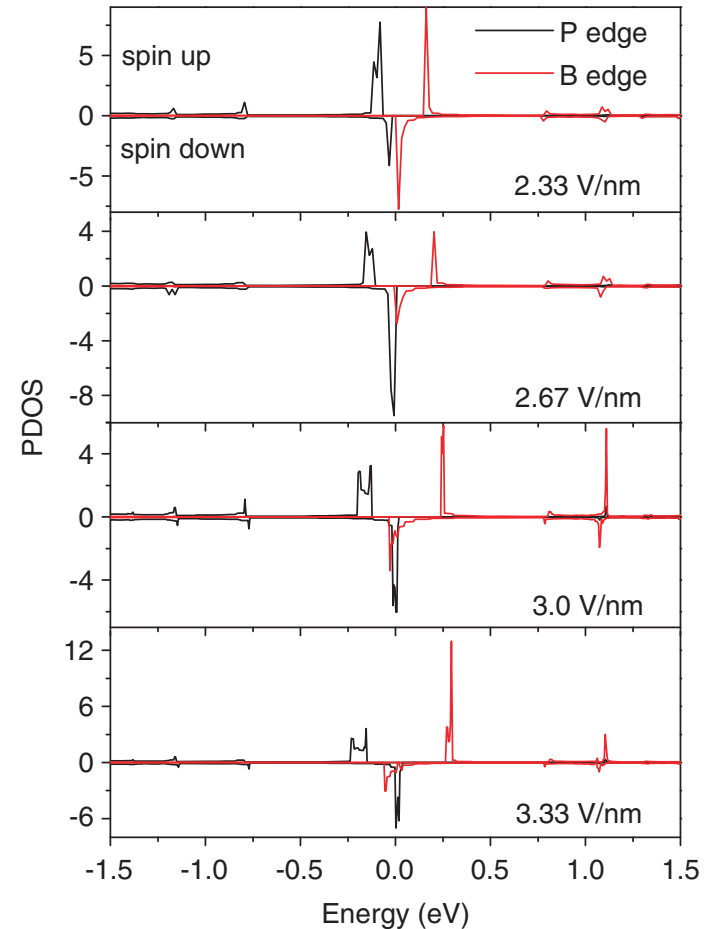

Figure 14 The PDOS on the $P(B)$ atoms at the $P(B)$ edge of the $8 z$ BPNR (zigzag boron phosphide nanoribbon) under different field strengths.

When the field strength was increased to $-3.33 \mathrm{~V} \mathrm{~nm}^{-1}$, the Bloch state of the HOVBM (LUCBM) was primarily localized at the P (B) atoms at the B (P) edge of the $8 z \mathrm{BPNR}$ (as shown in Figure $15 \mathrm{~d}$ ). Consequently, the bandgap of the $8 z \mathrm{BPNR}$ first increased and then decreased as the negative transverse electric field strengthened. In addition, after the Bloch state of the HOVBM (LUCBM) reached the $\mathrm{B}(\mathrm{P})$ edge of the $8 z \mathrm{BPNR}$, the bandgap began to decrease linearly, which was primarily dominated by the electrostatic potential difference between the $\mathrm{B}$ and $\mathrm{P}$ edges. Furthermore, during this process, the $8 z$ BPNR exhibited a change from a direct to an indirect bandgap. Compared with $z$ BNNRs and $z \mathrm{GNRs}$, the $z$ BPNRs exhibited versatile responses to the transverse electric field. This property may make $z$ BPNRs promising building blocks for multi-functional nanoelectronics.

Note that the free-electron-like states above the Fermi energy level may have a profound effect on the electronic structure of $z$ BPNRs under strong transverse electric fields. From the change in the band structure of the 8zBPNR shown in Figure 15, it can be predicted that if the transverse electric field is strong enough, the free-electron-like states would approach the Fermi energy and cause both the spin-up and the spin-down bandgaps to close. This prediction was verified by the variation in the bandgap variation of the $6 z$ BPNR shown in Figure 13d, where the free-electron-like states began to qualitatively change the electronic structure of the $6 z \mathrm{BPNR}$ at the field strength of -3.0 and $4.0 \mathrm{Vnm}^{-1}$. $\mathrm{N}$ atoms have the same valence electrons as $\mathrm{P}$ atoms, implying that $z \mathrm{BNNRs}$ may have similar properties to $z$ BPNRs. Therefore, it is necessary to investigate the spin-polarized electronic structures of $z$ BNNRs to compare with the spin-unpolarized DFT calculations performed by Park and Louie ${ }^{26}$ and Zhang and Guo. ${ }^{27}$ Figures $16 \mathrm{a}$ and $\mathrm{b}$ show the spin-polarized band structures of the $8 z \mathrm{BNNR}$ under 3.67 and $4.67 \mathrm{~V} \mathrm{~nm}^{-1}$. In contrast to the $8 z \mathrm{BPNR}$, the free-electron-like states in the $8 z \mathrm{BNNR}$ move considerably faster 

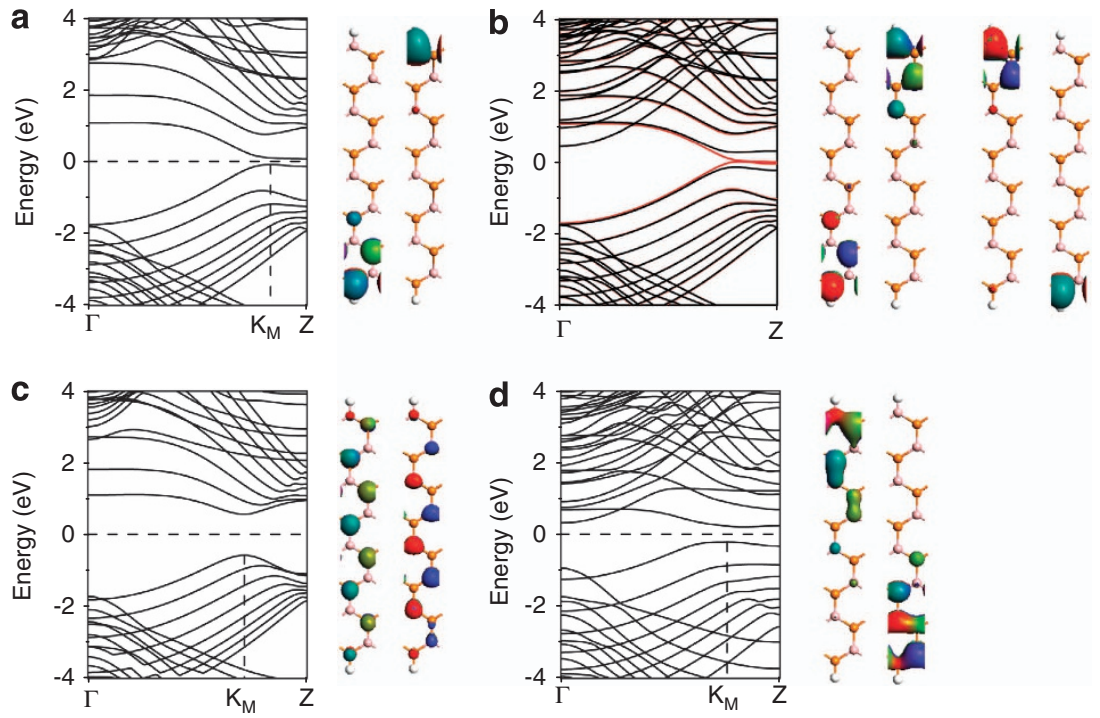

Figure 15 The band structures (left panel) and Bloch states (right panel) of the highest occupied valence band maximum (HOVBM) and the lowest unoccupied conductance band minimum (LUCBM) of the 8zBPNR (zigzag boron phosphide nanoribbon) under transverse electric fields of 1.67 (a), 3.33 (b), -0.67 (c) and $-3.33 \mathrm{Vnm}^{-1}$ (d). (Black and red lines denote spin up and spin down, respectively. The Bloch states of the HOVBM and LUCBM are shown from the left to right).
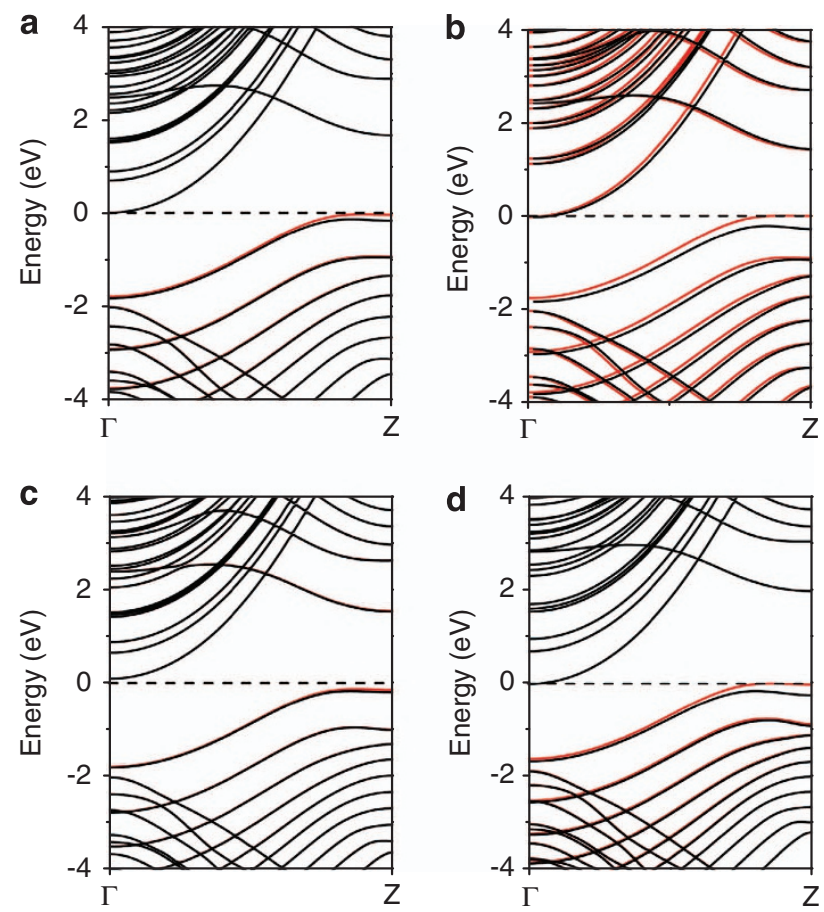

Figure 16 The band structures of the 8zBNNR (zigzag boron nitride nanoribbon) under transverse electric fields of (a) 3.67 and (b) $4.67 \mathrm{Vnm}^{-1}$, and the band structures of the 10zBNNR under transverse electric fields of (c) 3.0 and (d) $3.5 \mathrm{~V} \mathrm{~nm}^{-1}$.

than its edge states, changing the $8 z B N N R$ to be metallic. This finding is consistent with Park and Louie ${ }^{26}$ and Zhang and Guo's ${ }^{27}$ results. The $8 z$ BNNR actually exhibited magnetic properties under a field strength of 3.67 and $4.67 \mathrm{~V} \mathrm{~nm}^{-1}$, but this property is too weak to be applicable. Therefore, $z$ BPNRs may have advantages over $z$ BNNRs in spintronic applications. The $10 z \mathrm{BNNR}$, which is wider than the
$8 z \mathrm{BNNR}$, was studied for further verification. As shown in Figures $16 \mathrm{c}$ and $\mathrm{d}$, the $10 z \mathrm{BNNR}$ responded to the transverse electric field in the same way as the $8 z \mathrm{BNNR}$, except for the weaker field strength required because its ribbon width was wider than that of the $8 z$ BNNR.

\section{CONCLUSION}

The electronic properties and potential applications of nano-structures constructed using $z$ BPNRs and $z$ SiCNRs were revealed using first-principles DFT and non-equilibrium Green function calculations. $z$ BPNRs were found to be non-magnetic direct bandgap semiconductors with bandgaps of $\sim 1 \mathrm{eV}$. Owing to the transfer of electrons from the $\mathrm{P}$ to the $\mathrm{B}$ atoms and to the edge states, the bandgaps of the $z$ BPNRs decreased to levels below that of the hexagonal BP sheet as the ribbon width increased. A hybrid structure composed of a $z$ BPNR connected between two $z$ SiCNRs was proposed for constructing multi-functional nano-devices. For two-probe $z \mathrm{SiC}-\mathrm{BP}-\mathrm{SiC}$ devices, NDR resulting from the change of SiC-BP coupling under an applied bias was observed, and this NDR can be modulated by changing the $z$ BPNR length. In addition, significant electron transmission energy gaps of $\sim 1 \mathrm{eV}$ were observed in these hybrid structures. We also constructed ' $\mathrm{Y}$ '-, 'O'- and ' $\Delta$ '-shaped nano-structures using $z$ SiCNRs and $z$ BPNRs. The ' $\mathrm{Y}$ '- and ' $\Delta$ '-shaped structures were observed to exhibit significant spin polarization characteristics at the edges, making them potential building blocks for spintronics. Moreover, a transverse electric field can induce the $z$ BPNRs to change from nonmagnetic direct bandgap semiconductors to non-magnetic indirect bandgap semiconductors, ferrimagnetic semiconductors or halfmetals, depending on the strength and direction of the field. The results of this study are applicable in designing multi-functional nano-electronics constructed from hybrid SiC-BP nano-structures and in understanding their electron transport properties.

\section{CONFLICT OF INTEREST}

The authors declare no conflict of interest. 


\section{ACKNOWLEDGEMENTS}

We would like to acknowledge the support from the National Natural Science Foundation of China (Grant no. 51271100). This work is also supported by the National Basic Research Program of China (Grant no. 2012CB825702).

1 Novoselov, K. S., Geim, A. K., Morozov, S. V., Jiang, D., Zhang, Y., Dubonos, S. V., Grigorieva, I. V. \& Firsov, A. A. Electric field effect in atomically thin carbon films. Science 306, 666-669 (2004)

2 Castro Neto, A. H., Guinea, F., Peres, N. M. R., Novoselov, K. S. \& Geim, A. K. The electronic properties of graphene. Rev. Mod. Phys. 81, 109-162 (2009).

3 Chen, J. -H., Jang, C., Xiao, S., Ishigami, M. \& Fuhrer, M. S. Intrinsic and extrinsic performance limits of graphene devices on $\mathrm{SiO}_{2}$. Nat. Nanotech 3, 206-209 (2008).

4 Chen, F., Xia, J., Ferry, D. K. \& Tao, N. Dielectric screening enhanced performance in graphene FET. Nano Lett. 9, 2571-2574 (2009).

5 Frank, D. J., Taur, Y. \& Wong, H. -S. P. Generalized scale length for two-dimensional effects in MOSFETs. IEEE Electron Dev. Lett. 19, 385-387 (1998).

6 Liao, L., Bai, J. W., Qu, Y. Q., Lin, Y. C., Li, Y. J., Huang, Y. \& Duan, X. F. High-K oxide nanoribbons as gate dielectrics for high mobility topgated graphene transistors. Proc. Natl Acad. Sci. USA 107, 6711-6715 (2010).

7 Li, X., Cai, W. W., An, J. H., Kim, S. Y., Nah, J., Yang, D. X., Piner, R., Velamakanni, A., Jung, I., Tutuc, E., Banerjee, S. K., Colombo, L. \& Ruoff, R. S. Large-area synthesis of high-quality and uniform graphene films on copper foils. Science 324, 1312-1314 (2009).

8 Li, X., Wang, X., Zhang, L., Lee, S. \& Dai, H. Chemically derived, ultrasmooth graphene nanoribbon semiconductors. Science 319, 1229-1232 (2008).

9 Son, Y. W., Cohen, M. L. \& Louie, S. G. Energy gaps in graphene nanoribbons. Phys. Rev. Lett. 97, 216803-216806 (2006).

10 Hicks, J., Tejeda, A., Taleb-Ibrahimi, A., Nevius, M. S., Wang, F., Shepperd, K., Palmer, J., Bertran, F., Le Fèvre, P., Kunc, J., de Heer, W. A., Berger, C. \& Conrad, E. H. A widebandgap metal-semiconductor-metal nanostructure made entirely from graphene. Nat. Phys. 9, 49-54 (2013).

11 Sofo, J. O., Chaudhari, A. S. \& Barber, G. D. Graphane: A two-dimensional hydrocarbon. Phys. Rev. B 75, 153401 (2007).

12 Gan, L., Zhou, J., Ke, F., Gu, H., Li, D. N., Hu, Z. H., Sun, Q. \& Guo, X. F. Tuning the properties of graphene using a reversible gas-phase reaction. NPG Asia Mater. 4, e31. doi:10.1038/am.2012.58 (2012).

13 Ci, L. J., Song, L., Jin, C. h. H., Jariwala, D., Wu, D. X., Li, Y. J., Srivastava, A., Wang, Z. F., Storr, K., Balicas, L., Liu, F. \& Ajayan, P. M. Atomic layers of hybridized boron nitride and graphene domains. Nat. mater 9, 430-435 (2010).

14 Radisavljevic, B., Radenovic, A., Brivio1, J., Giacometti, V. \& Kis, A. Single-layer MoS transistors. Nat. Nanotech. 6, 147-150 (2011)

15 Golberg, D., Bando, Y., Huang, Y., Terao, T., Mitome, M., Tang, C. C. \& Zhi, C. Y. Boron nitride nanotubes and nanosheets. ACS Nano 4, 2979-2993 (2010).

16 Quhe, R., Zheng, J. X., Luo, G. F., Liu, Q. H., Qin, R., Zhou, J., Yu, D. P., Nagase, S., Mei, W. N., Gao, Z. X. \& Lu, J. Tunable and sizable band gap of single-layer graphene sandwiched between hexagonal boron nitride. NPG Asia Mater 4, e6. doi:10.1038/ am.2012.10 (2012).

17 Ferreira, G. J., Leuenberger, M. N. Loss, D. \& Carlos Egues, J. Low-bias negative differential resistance in graphene nanoribbon superlattices. Phys. Rev. B 84, 125453 (2011).

18 An, Y. P. \& Yang, Z. Q. Abnormal electronic transport and negative differential resistance of graphene nanoribbons with defects. Appl. Phys. Lett. 99, 192102 (2011).

19 Li, X. ,F., Wang, L. L., Chen, K. Q. \& Luo, Y. Strong current polarization and negative differential resistance in chiral graphene nanoribbons with reconstructed $(2,1)$-edges. Appl. Phys. Lett. 101, 073101-073105 (2012).
20 Wang, M. \& Li, C. M. Negative differential resistance in oxidized zigzag graphene nanoribbons. Phys. Chem. Chem. Phys. 13, 1413-1418 (2011).

21 Dragoman, D. \& Dragoman, M. Negative differential resistance of electrons in graphene barrier. Appl. Phys. Lett. 90, 143111 (2007)

22 Saha, K. K., Blom, A., Thygesen, K. S. \& Nikolić, B. K. Magnetoresistance and negative differential resistance in $\mathrm{Ni} /$ graphene/ $\mathrm{Ni}$ vertical heterostructures driven by finite bias voltage: a first-principles study. Phys. Rev. B 85, 184426 (2012).

23 Cheraghchi, H. \& Esfarjani, K. Negative differential resistance in molecular junctions: application to graphene ribbon junctions. Phys. Rev. B 78, 085123 (2008).

24 Wu, Y. Q., Farmer, D. B., Zhu, W. J., Han, S. J., Dimitrakopoulos, C. D., Bol, A. A., Avouris, P. \& Lin, Y. M. Three-terminal graphene negative differential resistance devices. ACS Nano 6, 2610-2616 (2012).

25 Son, Y. W., Cohen, M. L. \& Louie, S. G. Half-metallic graphene nanoribbons. Nature 444, 347-349 (2003)

26 Park, C. H. \& Louie, S. G. Energy gaps and stark effect in boron nitride nanoribbons. Nano Lett. 8, 2200-2203 (2008).

27 Zhang, Z. H. \& Guo, W. L. Energy-gap modulation of BN ribbons by transverse electric fields: first-principles calculations. Phys. Rev. B 77, 075403 (2008).

28 Sun, L., Li, Y. F., Li, Z. Y., Li, Q. X., Zhou, Z., Chen, Z. F., Yang, J. L. \& Hou, J. G. Electronic structures of SiC nanoribbons. J. Chem. Phys. 129, 174114-174117 (2008).

29 Lou, P. \& Lee, J. Y. Band structures of narrow zigzag silicon carbon nanoribbons. J. Phys. Chem. C 113, 12637-12640 (2009).

30 Lou, P. \& Lee, J. Y. Electrical control of magnetization in narrow zigzag silicon carbon nanoribbons. J. Phys. Chem. C 113, 21213-21217 (2009).

31 Zheng, F. L., Zhang, Y., Zhang, J. M. \& Xu, K. W. Bandgap modulations of silicon carbon nanoribbons by transverse electric fields: a theoretical study. Phys. Status Solidi B 248, 1676-1681 (2011).

32 Dolui, K., Pemmaraju, C. D. \& Sanvito, S. Electric field effects on armchair $\mathrm{MoS}_{2}$ nanoribbons. ACS Nano 6, 4823-4834 (2012).

33 Alejandro, L. C. Prediction of boron-phosphorous nanographene-like material. Int. J. Quantum Chem. 112, 3152-3157 (2012).

34 Brandbyge, M., Mozos, J. L., Ordejon, P., Taylor, J. \& Stokbro, K. Density-functional method for nonequilibrium electron transport. Phys. Rev. B. 65, 165401-165417 (2002).

35 ATK. QuantumWise. Copenhagen, Denmark (2008) www.quantumwise.com

$36 \mathrm{Li}$, Z. Y., Qian, H. Y., Wu, J., Gu, B. L. \& Duan, W. H. Role of symmetry in the transport properties of graphene nanoribbons under bias. Phys. Rev. Lett. 100, 206802 (2008)

$37 \mathrm{Lu}$, W. C., Meunier, V. \& Bernholc, J. Nonequilibrium quantum transport properties of organic molecules on silicon. Phys. Rev. Lett. 95, 206805 (2005).

38 Qi, J. S., Huang, J. Y., Feng, J., Shi, D. N. \& Li, J. The possibility of chemically inert, graphene-based all-carbon electronic devices with 0.8 eV gap. ACS Nano 5, 3475-3482 (2011).

39 Zheng, X. H., Lu, W. C., Abtew, T. A., Meunier, V. \& Bernholc, J. Negative differential resistance in $\mathrm{C}_{60}$-based electronic devices. ACS Nano 4, 7205-7210 (2010).

40 Zhu, L. Y., Wang, J. L., Zhang, T. T., Ma, L., Lim, C. W., Ding, F. \& Zeng, X. C. Mechanically robust tri-wing graphene nanoribbons with tunable electronic and magnetic properties. Nano Lett. 10, 494-498 (2010).

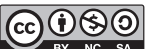

This work is licensed under a Creative Commons Attribution-NonCommercial-ShareAlike 3.0 Unported License. To view a copy of this license, visit http://creativecommons. org/licenses/by-nc-sa/3.0/ 